\title{
Probable factors affecting clinical outcomes of internal jugular vein stenosis
}

\author{
Chaobo Bai ${ }^{1,2,3}$, Zhongao Wang ${ }^{1,2,3}$, Jingwei Guan ${ }^{1,2,3}$, Kexin Jin ${ }^{1,2,3}$, Jingkun Sun ${ }^{1,2,3}$, Yuchuan Ding ${ }^{3,4}$, \\ Xunming $\mathrm{Ji}^{3,4,5}$, Ran Meng ${ }^{1,2,3}$
}

${ }^{1}$ Department of Neurology, Xuanwu Hospital, Capital Medical University, Beijing 100053, China; ${ }^{2}$ Advanced Center of Stroke, Beijing Institute for Brain Disorders, Beijing 100069, China; ${ }^{3}$ Department of China-America Institute of Neuroscience, Xuanwu Hospital, Capital Medical University, Beijing 100053, China; ${ }^{4}$ Department of Neurosurgery, Wayne State University School of Medicine, Detroit, MI, USA; ${ }^{5}$ Department of Neurosurgery, Xuanwu Hospital, Capital Medical University, Beijing 100053, China

Contributions: (I) Conception and design: C Bai, R Meng; (II) Administrative support: X Ji, Y Ding, R Meng; (III) Provision of study materials or patients: C Bai, Z Wang, J Guan, K Jin, J Sun; (IV) Collection and assembly of data: C Bai, Z Wang, J Guan, K Jin, J Sun; (V) Data analysis and interpretation: C Bai, R Meng; (VI) Manuscript writing: All authors; (VII) Final approval of manuscript: All authors.

Correspondence to: Ran Meng, MD, PhD. Department of Neurology, Xuanwu Hospital, Capital Medical University, Beijing 100053, China.

Email: victor65@126.com.

Background: Internal jugular vein stenosis (IJVS) has recently aroused increasing interests, whereas, the factors affecting its clinical outcomes are not clear. This study aims to explore the probable factors affected clinical prognosis by evaluating the IJVS with different etiologies and strategies.

Methods: Patients with IJVS confirmed by contrast-enhanced magnetic resonance venography (CEMRV) were enrolled from October 2017 through October 2018. One-year clinical outcomes of the IJVS cases enrolled in this study were assessed by outpatient and telephone follow-up using the Patient Global Impression of Change (PGIC) scores. According to the etiologies, patients were divided into thrombotic IJVS and non-thrombotic IJVS groups. And further, non-thrombotic IJVS group was divided into external compression and non-external compression subgroups. Outcomes of IJVS with different etiologies and strategies were compared and the probable prognostic factors were analyzed.

Results: A total of 118 eligible patients enrolled in this study, including 76 females and 42 males, mean aged 55.07 \pm 14.61 years. The average follow-up duration after discharge was $13.22 \pm 3.80$ months. According to the PGIC scores, we categorized patients as good outcome and poor outcome groups. For thrombotic IJVS, patients underwent standard anticoagulant obtained remarkable PGIC improvement (100.0\% vs. $33.3 \%, \mathrm{P}=0.038)$. For non-thrombotic IJVS, stenting showed benefit in non-external compression subgroup ( $26.9 \%$ vs. $3.3 \%, \mathrm{P}=0.019)$ but not in external compression subgroup. In addition, we found that in this Chinese IJVS cohort, poor outcomes involved old age $(\mathrm{P}=0.004)$, type 2 diabetes mellitus $(\mathrm{P}=0.036)$, previous hepatitis $\mathrm{B}$ virus $(\mathrm{HBV})$ infection $(\mathrm{P}=0.027)$, and head noises $(\mathrm{P}=0.002)$. Multivariate logistic regression analysis indicated that continuous head noises $[\mathrm{P}=0.045$, odds ratio $(\mathrm{OR}): 2.412,95 \%$ confidence interval (CI): 1.019-5.711], as a unique symptom of IJVS may be significantly related to poor outcomes.

Conclusions: In this Chinese cohort, elderly degenerative bone compression, type 2 diabetes mellitus, and previous $\mathrm{HBV}$ infection are the top-three probable etiologies of non-thrombotic IJVS and may involve poor outcome. Long-term head noises may predict IJVS and with poor outcome. Thrombosis-induced IJVS may get benefit from standard anticoagulation. Non-external compression IJVS can be corrected by stenting.

Keywords: Internal jugular vein stenosis (IJVS); etiology; contrast-enhanced magnetic resonance venography (CE-MRV); outcome

Submitted Aug 15, 2019. Accepted for publication Oct 25, 2019.

doi: $10.21037 / \mathrm{atm} .2019 .11 .05$

View this article at: http://dx.doi.org/10.21037/atm.2019.11.05 


\section{Introduction}

Recently, the internal jugular vein stenosis (IJVS) has aroused widespread attention. The internal jugular vein (IJV), as a continuation of the sigmoid sinus, is an important buffer zone between cerebral veins and central veins (1). However, due to the lack of smooth muscle, the IJV is more anatomically affected by the surrounding abnormal structures such as the internal carotid artery, adjacent bony structures, enlarged lymph nodes or thyroid, and sternocleidomastoid muscle (2). Besides, intraluminal anomalous factors, as well as systemic diseases, may also induce venous wall damage resulting in stenosis (3). Accordingly, the IJVS may cause cerebral venous outflow obstruction and hinder cerebral blood circulation, leading to a series of non-specific clinical symptoms, such as headache, tinnitus, head noises, visual disorders, hearing loss, memory loss, sleep disorders, and neck discomfort (4). In addition, the previous cohort study has indicated that stenting may be a promising treatment strategy to relieve some severe intracranial hypertension induced by non-thrombotic IJVS (5).

To the best of our knowledge, the majority of prior studies were mainly focused on discussing the relationship between IJVS and central nervous system (CNS) disorders (6-9). Few of them reported the clinical prognosis of IJVS. Given the current facts that the guidelines and consensus on IJVS are still lacking, this study aims to compare different treatment strategies on the prognosis and analyze the probable prognostic factors of IJVS, which may enhance the understanding to IJVS and avoid clinical misdiagnosis and treatment delay.

\section{Methods}

\section{Study design and patient's selection}

This single-center real-world study was conducted between October 2017 and October 2018 in Neurology Department, Xuanwu Hospital, Capital Medical University, Beijing, China, and was approved by the Ethics Committee of Xuanwu Hospital, Capital Medical University. All participants signed the consent forms before enrolment.

Patients were enrolled into this study based on the following included criteria: (I) patients with unilateral or bilateral IJVS as well as abnormally distorted collaterals surrounded, confirmed by contrast-enhanced magnetic resonance venography (CE-MRV) of the brain and neck were eligible for this study; (II) patient presented sustained or aggravated clinical symptoms: headache, tinnitus, head noises, visual disorders, hearing loss, memory loss, sleep disorders, and neck discomfort; (III) the IJVS diagnosis was made by our institution for the first time; (IV) no age, gender and comorbidities limitation.

Patients were excluded as the following criteria: (I) there were no abnormally distorted collaterals surrounded the stenotic IJV; (II) pre-existing ophthalmology or otology diseases; (III) CNS malignancy; (IV) patient with incomplete clinical data, such as contraindications to CEMRV, or lost the follow-up data.

\section{Blood and radiological assessment}

Blood routine items, blood biochemistry tests, and hepatitis $B$ virus (HBV) were assessed at admission. Previous HBV infection was defined as negative of hepatitis B surface antigen (HBsAg), and positive of hepatitis B core antibody (anti-HBc) and/or hepatitis B surface antibody (HBsAb) (10).

Imaging examination, including CE-MRV/black-blood MRI, and three-dimensional reconstruction images of cervical CT were applied to identify IJVS, IJV thrombosis, cerebral venous sinus thrombosis (CVST) and extraluminal compression assessment, respectively (11). All images were assessed completed by two experienced radiologists.

\section{Follow-up procedure}

We applied the Patient Global Impression of Change (PGIC) score to assess the 1-year outcomes of the patients by outpatient and telephone follow-up. The PGIC score is a semi-quantitated self-evaluation of the patients to their overall change of the symptoms using a 7 -point scale $(1=$ very much improved, $2=$ much improved, $3=$ minimally improved, $4=$ no change, $5=$ minimally worse, $6=$ much worse, $7=$ very much worse) (12). According to PGIC scores, we divided patients into two groups: good outcome group (PGIC scores $\leq 3$ ) and poor outcome group (PGIC >3).

We categorized patients as thrombotic IJVS and nonthrombotic IJVS groups. In non-thrombotic IJVS group, we further divided patients into non-external compression and external compression subgroups according to the different etiologies, and we evaluated the impact of different treatment strategies on the prognosis based on PGIC scores among the groups and subgroups, in an attempt to analyze the factors that influence the clinical outcomes of IJVS.

\section{Statistical analysis}

All data analysis for this study was performed by the Social 
Table 1 Baseline demographic data

\begin{tabular}{|c|c|}
\hline Clinical characteristics & Total $(n=118)$ \\
\hline \multicolumn{2}{|l|}{ Personal data } \\
\hline Age, years & $55.07 \pm 14.61$ \\
\hline Gender (male/female) & $42 / 76$ \\
\hline Time from onset to door, years & $4(1.5-10)$ \\
\hline Time from discharge to follow up, months & $13.22 \pm 3.80$ \\
\hline \multicolumn{2}{|l|}{ Clinical manifestations, $\mathrm{n}(\%)$} \\
\hline Headache & $59(50.0)$ \\
\hline Tinnitus & $66(55.9)$ \\
\hline Head noises & $74(62.7)$ \\
\hline Visual disorders & $37(31.4)$ \\
\hline Sleeping disorders & $80(67.8)$ \\
\hline Hearing loss & $39(33.1)$ \\
\hline Neck discomfort & $41(34.7)$ \\
\hline Memory loss & $12(10.2)$ \\
\hline \multicolumn{2}{|l|}{ Comorbidities, n (\%) } \\
\hline CVST & $13(11.0)$ \\
\hline Non-thrombotic CVSS & $13(11.0)$ \\
\hline Type 2 diabetes mellitus & $13(11.0)$ \\
\hline Hypertension & $40(33.9)$ \\
\hline Hyperlipemia & $51(43.2)$ \\
\hline Hyperhomocysteinemia & $8(6.8)$ \\
\hline Hyperuricemia & $10(8.5)$ \\
\hline Previous HBV infection & $56(47.5)$ \\
\hline \multicolumn{2}{|l|}{ Lifestyle habits, n (\%) } \\
\hline Smoking & $15(12.7)$ \\
\hline Alcohol drinking & $15(12.7)$ \\
\hline Osseous compression & $39(33.1)$ \\
\hline Arterial compression & $14(11.9)$ \\
\hline Lymph node compression & $1(0.8)$ \\
\hline
\end{tabular}

CVST, cerebral venous sinus thrombosis; CVSS, cerebral venous sinus stenosis; HBV, hepatitis $B$ virus.

Science Statistical Software Package (SPSS) version 21.0 program. The categorical variables were expressed as counts and percentages, continuous variables were calculated as mean \pm standard deviation, and discrete data was represented as median (interquartile range). Fisher's exact test is used to analyze categorical variables, and the Student $t$-test is applied for continuous variables. Mann-Whitney $\mathrm{U}$ test is applicable for data that are not normally distributed. In addition, multiple logistic regressions were used to analyze probable prognostic factors of IJVS. Two-sided $\mathrm{P}$ values $<0.05$ were considered a statistically significant difference.

\section{Results}

Table 1 shows the characteristics of IJVS cases. A total of 118 patients were enrolled, the mean age was $55.07 \pm 14.61$ years, and female $v s$. male was $76 v s$. 42 . The median time from symptoms onset to door of clinic was 4 years. The average follow-up duration after discharge was $13.22 \pm 3.80$ months. Clinical manifestation of IJVS are various, the topthree included head noises (62.7\%), tinnitus (55.9\%) and sleeping disorders $(67.8 \%)$. Others involved headache (50.0\%), visual disorders (31.4\%), hearing loss (33.1\%), neck discomfort (34.7\%), and memory loss (10.2\%). In addition, we analyzed comorbidities that may cause or affect IJVS, comprised CVST (11.0\%), non-thrombotic cerebral venous sinus stenosis (CVSS) (11.0\%), type 2 diabetes mellitus (11.0\%), hypertension (33.9\%), hyperlipemia (43.2\%), hyperhomocysteinemia (6.8\%), hyperuricemia (8.5\%), previous HBV infection (47.5\%). In our Chinese cohort, none of our patients were accompanied by multiple sclerosis. For lifestyle habits, in which $12.7 \%$ reported smoking and $12.7 \%$ have alcohol abuse. For abnormal anatomical structures around IJV, the largest proportion was osseous compression (33.1\%), followed by arterial compression (11.9\%) and lymph node compression (0.8\%).

We divided the patients into the thrombotic IJVS and the non-thrombotic IJVS groups according to the etiologies. In addition, according to PGIC scores, we divided the patients into good (PGIC scores $\leq 3$ ) and poor outcome groups (PGIC >3). A total of 13 patients were thrombotic IJVS, in which patients underwent anticoagulation had remarkable improvements on PGIC scores $(100.0 \%$ vs. $33.3 \%, \mathrm{P}=0.038)$. In addition, in the external compressioninduced non-thrombotic IJVS subgroup, stenting did not differ significantly in prognosis $(14.3 \%$ vs. $14.3 \%, \mathrm{P}=1.000)$. However, in the non-external compression subgroup, stenting showed great benefit $(26.9 \%$ vs. $3.3 \%, \mathrm{P}=0.019)$ (Table 2).

Comparison of the different factors on prognosis was shown in Table 3. In which, we found that older age $(\mathrm{P}=0.004)$, head noises $(\mathrm{P}=0.002)$, type 2 diabetes mellitus 
Table 2 Different treatment strategies in good and poor outcome groups of IJVS

\begin{tabular}{lcc}
\hline Treatment strategies & Good outcome & Poor outcome \\
\hline Thrombotic IJVS $(\mathrm{n}=13)$ & $10(100.0)$ & $1(33.3)$ \\
Standard anticoagulation, $\mathrm{n}(\%)$ & & $0.038^{\star}$ \\
Non-thrombotic IJVS $(\mathrm{n}=105)$ & & $4(14.3)$ \\
External compression $(\mathrm{n}=49)$ & $3(14.3)$ & 1.000 \\
$\quad$ Stenting, $\mathrm{n}(\%)$ & & $1(3.3)$ \\
Non-external compression $(\mathrm{n}=56)$ & $7(26.9)$ & $0.019^{*}$ \\
\hline
\end{tabular}

*, statistically significant at $\mathrm{P}<0.05$. IJVS, internal jugular vein stenosis.

Table 3 Different factors between good and poor outcomes of IJVS

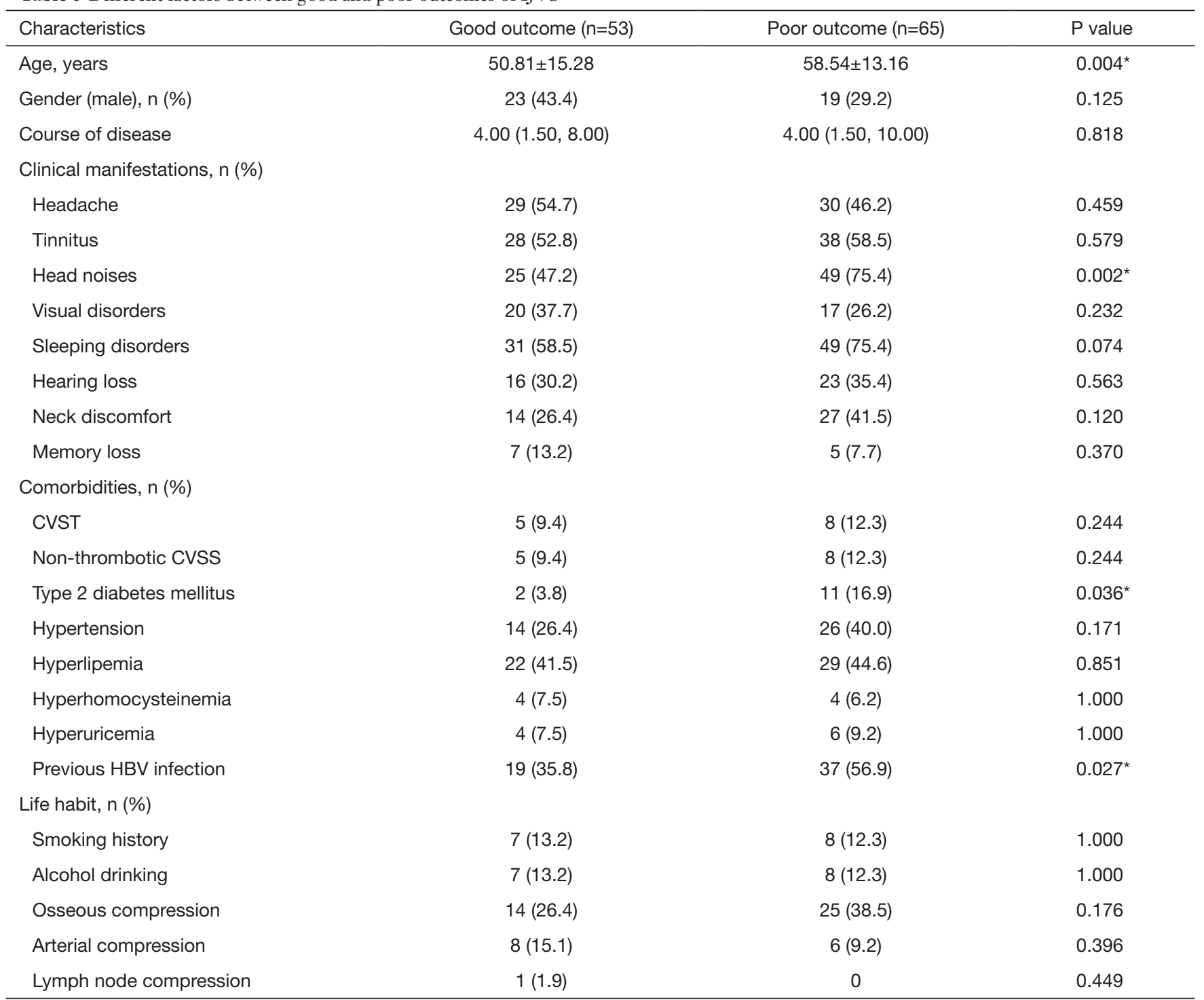

*, statistically significant at $\mathrm{P}<0.05$. IJVS, internal jugular vein stenosis; CVST, cerebral venous sinus thrombosis; CVSS, cerebral venous sinus stenosis; HBV, hepatitis $B$ virus. 
Table 4 Multivariate logistic regression analysis of factors for poor outcomes

\begin{tabular}{lccc}
\hline Variables & P value & OR value & $95 \% \mathrm{Cl}$ \\
\hline Age & 0.188 & 1.021 & $0.990-1.052$ \\
Head noises & $0.045^{\star}$ & 2.412 & $1.019-5.711$ \\
Type 2 diabetes mellitus & 0.131 & 3.483 & $0.690-17.581$ \\
Previous HBV infection & 0.277 & 1.575 & $0.694-3.576$ \\
\hline
\end{tabular}

*, statistically significant at $\mathrm{P}<0.05$. $\mathrm{HBV}$, hepatitis $\mathrm{B}$ virus; $\mathrm{OR}$, odds ratio; $\mathrm{Cl}$, confidence interval.

$(\mathrm{P}=0.036)$, previous $\mathrm{HBV}$ infection $(\mathrm{P}=0.027)$ were significantly associated with poor outcome in this Chinese IJVS cohort. In addition, multivariate logistic regression analysis indicates that head noises $[\mathrm{P}=0.045$, odds ratio $(\mathrm{OR})$ : 2.412, 95\% confidence interval (CI): 1.019-5.711] may significantly related to poor outcome in IJVS cohort (Table 4).

\section{Discussion}

Recently, IJVS as a chronic venous outflow insufficiency, has gained increasing attention $(6-8,13)$. In previous studies, most of the patients were confirmed the diagnosis at several years after the onset of the symptoms. Clinical symptoms such as headache, tinnitus, head noises, visual disorders, memory loss, sleep disorders, and neck discomfort, may continuously impair the patient's quality of life but lack the specificity and focal signs. Therefore, lacking understanding of the disease and high misdiagnosis rate of clinicians may lead to treatment delay.

It is known that various factors may relate to IJVS, such as extrinsic structure compression of IJV, intraluminal anomalies, and systemic disease-related IJV wall anomalies mediated stenosis $(1-3,5)$. Our study found that bone compression $(33.1 \%)$ is the most common external compression factor in this Chinese cohort. Whereas, poorly controlled diabetes, previous HBV infection, thrombi and long-term alcohol use may contribute to damage of the IJV wall resulting in IJVS $(1,14)$. In this Chinese cohort, none of our patients were accompanied by multiple sclerosis. However, IJVS was frequently reported accompanied by multiple sclerosis in Caucasian (15). Accordingly, the etiologies of IJVS in native Chinese may differ from Caucasian, which may have their own characteristic.

\section{Different treatment strategies on the prognosis of IFVS}

According to the etiologies, IJVS in this cohort can be divided into thrombotic and non-thrombotic. In 13 patients with thrombotic IJVS, their PGIC improved remarkably after underwent standard anticoagulation. Anticoagulant therapies may inhibit thrombus progressing, as well as promoting revascularization of $\operatorname{IJV}(1,16)$. Meanwhile, none spontaneous intracranial hemorrhage occurred in this cohort. So, thrombotic IJVS may get benefit from anticoagulation, however more solid evidence about its safety and efficacy is still needed in further clinical practice.

Severe CVSS and IJVS can induce intracranial hypertension, resulting in irreversible vision damage. Previous studies have indicated that timely stenting could effectively reduce intracranial pressure and prevent visual deterioration $(5,17-19)$. Our study revealed that stenting showed significant benefits in non-external compression groups. However, stenting alone did not resolve the IJVS induced by external compression. For non-thrombotic IJVS induced by external compression, decompression surgery combined with stenting may obtain good clinical outcome at pathological mechanisms level (20).

\section{Probable factors related to the clinical outcome of IfVS}

By comparing the different outcomes of the IJVS patients in this Chinese cohort, we found that older age, head noises, type 2 diabetes mellitus, and previous $\mathrm{HBV}$ infection were significantly associated with poor outcome. For elder patients, the ratio of external bone compression mediated IJVS was higher than other issues, presented as long styloid process or the cervical vertebrae hyperplasia that compressed bilateral or unilateral IJV, which were often misdiagnosed or missed the diagnosis in routine work previously, resulting in mistreatment or treatment delay (1).

Type 2 diabetes mellitus is a well-known risk factor for cerebral arterial disease, it can also damage the venous wall, resulted in IJVS by damaging the endothelium $(21,22)$. For patients with type 2 diabetes, strict glycemic control may be helpful for IJVS clinical outcomes, even though the evidence is still not enough, our study is ongoing. 
$\mathrm{HBV}$ infection is reported to be the leading cause of liver cirrhosis and liver cancer in China (23-26). HBV-mediated autoimmune responses may lead to liver vascular endothelial cell injury and hepatic vein stenosis, resulting in increased hepatic vascular resistance and portal hypertension. Wang et al. found that previous $\mathrm{HBV}$ infection significantly increased the risk of decompensated cirrhosis (23-26). Moreover, the damage of blood vessels caused by HBV is systemic (23-26). Whereby, it can be speculated that previous HBV infection may damage the vascular endothelial cells of IJV through chronic autoimmune impairment. However, this impairment may be chronically worsening if $\mathrm{HBV}$ infection is not effectively controlled. Therefore, IJVS with previous hepatitis B infection may experience chronic viral-mediated endothelial damage, which may affect the clinical outcomes of IJVS. However, these speculations require more clinical evidence.

In this study, we found that head noises may be associated with poor outcome of IJVS. Head noises are generally considered to be significant hemodynamic alterations for IJVS $(1,5)$. It is worth noting that some patients with IJVS did not exhibit apparent clinical symptoms despite noticeable stenosis was identified by CE-MRV. This may be due to the vertebral venous plexus around the stenosis is well compensated or the contralateral side is unobstructed. However, whether this significant hemodynamic change is the result of poor vertebral venous plexus compensation around IJVS is still unclear. In addition, head noises are often misdiagnosed as arterial or some other disorders in the clinic initially, which may result in IJVS treatment delay and poor outcome.

This study has several limitations. Firstly, the duration of follow-up is not long enough. Secondly, the PGIC score is the subjective feelings of IJVS patients. Future studies require more accurate scoring to assess clinical symptoms. Finally, our study is a single-center study enrolled only Chinese IJVS cohort and the case number are not large enough. Multi-center study with more cases is still needed in the future.

\section{Conclusions}

In this Chinese cohort, elderly degenerative bone compression, type 2 diabetes mellitus, and previous HBV infection are the top-three probable etiologies of nonthrombotic IJVS and may involve poor outcome. Longterm head noises may predict IJVS and with poor outcome. Thrombosis-induced IJVS may get benefit from standard anticoagulation. Non-external compression IJVS can be corrected by stenting.

\section{Acknowledgments}

We would like to thank all patients and doctors who participated in this study for their cooperation.

Funding: This study was sponsored by the National Key R\&D Program of China (2017YFC1308401), the National Natural Science Foundation (81371289), the Project of Beijing Municipal Top Talent for Healthy Work of China (2014-2-015).

\section{Footnote}

Conflicts of Interest: The authors have no conflicts of interest to declare.

Ethical Statement: The authors are accountable for all aspects of the work in ensuring that questions related to the accuracy or integrity of any part of the work are appropriately investigated and resolved. This study was approved by the Ethics Committee of Xuanwu Hospital, Capital Medical University (No. 2017-013). All participants signed the consent forms before enrolment.

\section{References}

1. Zhou D, Ding JY, Ya JY, et al. Understanding jugular venous outflow disturbance. CNS Neurosci Ther 2018;24:473-82.

2. Ding JY, Zhou D, Pan LQ, et al. Cervical spondylotic internal jugular venous compression syndrome. CNS Neurosci Ther 2019. [Epub ahead of print].

3. Zhou D, Ding J, Asmaro K, et al. Clinical characteristics and neuroimaging findings in internal jugular venous outflow disturbance. Thromb Haemost 2019;119:308-18.

4. Bai C, Xu Y, Zhou D, et al. The comparative analysis of non-thrombotic internal jugular vein stenosis and cerebral venous sinus stenosis. J Thromb Thrombolysis 2019;48:61-7.

5. Zhou D, Meng R, Zhang X, et al. Intracranial hypertension induced by internal jugular vein stenosis can be resolved by stenting. Eur J Neurol 2018;25:365-e13.

6. Zamboni P, Galeotti R, Menegatti E, et al. Chronic cerebrospinal venous insufficiency in patients with multiple sclerosis. J Neurol Neurosurg Psychiatry 2009;80:392-9.

7. Chung CP, Beggs C, Wang PN, et al. Jugular venous reflux 
and white matter abnormalities in Alzheimer's disease: a pilot study. J Alzheimers Dis 2014;39:601-9.

8. Liu M, Xu H, Wang Y, et al. Patterns of chronic venous insufficiency in the dural sinuses and extracranial draining veins and their relationship with white matter hyperintensities for patients with Parkinson's disease. J Vasc Surg 2015;61:1511-20.e1.

9. Han K, Chao AC, Chang FC, et al. Obstruction of venous drainage linked to transient global amnesia. PLoS One 2015;10:e0132893.

10. Mandalà M, Fagiuoli S, Francisci D, et al. Hepatitis B in immunosuppressed cancer patients: pathogenesis, incidence and prophylaxis. Crit Rev Oncol Hematol 2013;87:12-27.

11. Yang Q, Duan J, Fan Z, et al. Early detection and quantification of cerebral venous thrombosis by magnetic resonance black-blood thrombus imaging. Stroke 2016;47:404-9.

12. Dworkin RH, Turk DC, Wyrwich KW, et al. Interpreting the clinical importance of treatment outcomes in chronic pain clinical trials: IMMPACT recommendations. J Pain 2008;9:105-21.

13. Bruno A, Napolitano M, Califano L, et al. The prevalence of chronic cerebrospinal venous insufficiency in Meniere disease: 24-month follow-up after angioplasty. J Vasc Interv Radiol 2017;28:388-91.

14. Zivadinov R, Chung CP. Potential involvement of the extracranial venous system in central nervous system disorders and aging. BMC Med 2013;11:260.

15. Zwischenberger BA, Beasley MM, Davenport DL, et al. Meta-analysis of the correlation between chronic cerebrospinal venous insufficiency and multiple sclerosis. Vasc Endovascular Surg 2013;47:620-4.

16. Saposnik G, Barinagarrementeria F, Brown RD Jr, et al. Diagnosis and management of cerebral venous thrombosis: a statement for healthcare professionals from the American Heart Association/American Stroke Association. Stroke
2011;42:1158-92.

17. Ahmed RM, Wilkinson M, Parker GD, et al. Transverse sinus stenting for idiopathic intracranial hypertension: a review of 52 patients and of model predictions. AJNR Am J Neuroradiol 2011;32:1408-14.

18. Radvany MG, Solomon D, Nijjar S, et al. Visual and neurological outcomes following endovascular stenting for pseudotumor cerebri associated with transverse sinus stenosis. J Neuroophthalmol 2013;33:117-22.

19. Shazly TA, Jadhav AP, Aghaebrahim A, et al. Venous sinus stenting shortens the duration of medical therapy for increased intracranial pressure secondary to venous sinus stenosis. J Neurointerv Surg 2018;10:310-4.

20. Higgins JN, Garnett MR, Pickard JD, et al. An evaluation of styloidectomy as an adjunct or alternative to jugular stenting in idiopathic intracranial hypertension and disturbances of cranial venous outflow. J Neurol Surg B Skull Base 2017;78:158-63.

21. Chung WS, Lin CL, Kao CH. Diabetes increases the risk of deep-vein thrombosis and pulmonary embolism. A population-based cohort study. Thromb Haemost 2015;114:812-8.

22. Usdan LS, Choong KW, McDonnell ME. Type 2 diabetes mellitus manifesting with a cerebral vein thrombosis and ketoacidosis. Endocr Pract 2007;13:687-90.

23. Wang FS, Fan JG, Zhang Z, et al. The global burden of liver disease: the major impact of China. Hepatology 2014;60:2099-108.

24. Ganem D, Prince AM. Hepatitis B virus infection-natural history and clinical consequences. $\mathrm{N}$ Engl J Med 2004;350:1118-29.

25. Bosch J. Vascular deterioration in cirrhosis: the big picture. J Clin Gastroenterol 2007;41 Suppl 3:S247-53.

26. Wang H, Swann R, Thomas E, et al. Impact of previous hepatitis $\mathrm{B}$ infection on the clinical outcomes from chronic hepatitis C? A population-level analysis. J Viral Hepat 2018;25:930-8.
Cite this article as: Bai C, Wang Z, Guan J, Jin K, Sun J, Ding Y, Ji X, Meng R. Probable factors affecting clinical outcomes of internal jugular vein stenosis. Ann Transl Med 2019;7(22):621. doi: 10.21037/atm.2019.11.05 